\title{
Using "IF-THEN" plans to increase physical activity
}

\section{Data analysis plan \\ Randomisation checks}

We will run tests on all outcome and demographic variables to determine if there are any baseline differences in any of these between conditions: chi square will be used for categorical variables and ANOVA for continuous variables.

\section{Hypothesis 1}

The two conditions that complete a volitional help sheet (standard VHS and single situation VHS) will report greater physical activity and lower sedentariness at follow up than the control condition

Three mixed ANCOVAs will be used with VHS condition as the between participants variable ( 3 levels: standard VHS, single situation and control) and time as the within participants variable (baseline and $6 \mathrm{~m}$ follow up). As age, gender and SES are related to level of physical activity these will be used as a covariate. The ANCOVAs will be conducted for the IPAQ (METS), and the sedentariness questionnaire. If there is a significant time $x$ condition interaction then we will use planned contrasts to determine if the conditions differ from each other in the predicted way.

A hierarchical logistic regression will also be conducted to determine if category of activity is significantly different at follow up between conditions. Category of activity (active or inactive calculated from the Sport England measure will be regressed onto condition (dummy coded: standard VHS, single situation and control); age, gender and SES will be controlled for at step 1.

\section{Hypothesis 2}

\section{Increases in physical activity/reductions in sedentariness will be associated with improved subjective well-being}

To account for changes in physical activity (IPAQ) and sedentariness affecting subjective wellbeing, we will use regression analyses to compute residual scores that will capture any changes between baseline and follow-up.

We will conduct a multiple regression analysis with changes in IPAQ and sedentariness as independent variables, and subjective well-being at follow-up as the dependent variable. We will control for VHS condition, age, gender and SES in these analyses. 


\section{Hypothesis 3}

The VHS interventions will be most effective for those who have developed high habits, awareness of standards, self-monitoring, self-regulatory effort, capabilities, opportunities and motivations to increase physical activity and are currently not meeting health behaviour guidelines (including physical activity, smoking, alcohol consumption and diet).

Two mediation analyses will be conducted using the Hayes mediation model in SPSS with VHS condition (2 levels: combined VHS versus control) as the independent variable, and (i) IPAQ and (ii) sedentariness as the dependent variables. We will use regression analyses to compute residual scores that will capture any changes between baseline and follow-up. Thus, changes in habits, self regulation (awareness of standards, self monitoring and self regulatory effort), and COM (capabilities, opportunities and motivations) will be tested as mediators. For each of these two mediation analyses SES, age and gender will be used as covariates.

To determine if physical activity, smoking, alcohol consumption and diet at baseline are moderators they will be dichotomised according to whether people are meeting UK government recommendations as opposed to not meeting UK government recommendations for each. For the IPAQ, a series of mixed ANCOVAs will be used with VHS condition (3 levels: standard VHS, single situation and control) and the moderators (e.g., meeting vs. not meeting each of the recommendations) as the between-participants variables and time as the withinparticipants variable (baseline and $6 \mathrm{~m}$ follow up). SES, age and gender will be used as covariates.

A multinomial hierarchical logistic regression will also be conducted to determine if category of activity is a moderator. Condition (dummy coded: standard VHS, single situation and control) will be regressed onto the dichotomised categories of meeting or not meeting recommendations for each of the health behaviours, Sport England categories (active or inactive) and the interaction term of each health behaviour and Sport England category. SES, age and gender will be entered at Step 1 to act as covariates. 


\section{Scoring the main outcome measures}

\section{Sport England Short Active Lives Survey (categorical variable):}

The final score is calculated by summing all activities as follows (only activities identified as increasing breathing rate are included):

(Days of walking * usual minutes of walking IF sufficient to raise breathing rate) + (Days of cycling * usual munities of cycling IF sufficient to raise breathing rate) + (days of sport, fitness or dance * usual minutes of sport, fitness or dance IF sufficient to raise breathing rate).

Any activities which were not identified as sufficient to increase breathing rate by the respondent are excluded from the calculation.

Anyone completing a total of less than 30 minutes activity sufficient to increase breathing rate is classified as 'inactive', anyone completing between 30 minutes and 149 minutes is classified as 'fairly active' and anyone completing $150+$ minutes is classified as 'active'

\section{IPAQ (continuous variable):}

Walking: multiply number of minutes by 3.3 and then multiply by number of days Moderate: multiply number of minutes by 4 and then multiply by number of days Vigorous: multiply number of minutes by 8 and then multiply by number of days

Sum the 3 numbers for a METS score.

\section{Sedentary behaviour questionnaire}

Reponses will be reported in continuous time, in hours and minutes per week. Weekly time in each activity will be converted to hours per day. Total sedentary time will be calculated as the sum of daily time in each activity and reported as hours per day.

\section{Self-regulation}

Mean of the two items for each of three sub-constructs: awareness of standards, selfmonitoring, and self regulatory effort.

\section{COM (capabilities, opportunities and motivations)}

Each of the items will be analysed separately as these each measure a separate construct. 


\section{Habits}

Mean of the four items. 